\title{
CLINICOPATHOLOGICAL CORRELATION OF LIVER BIOPSY- STUDY OF 50 CASES
}

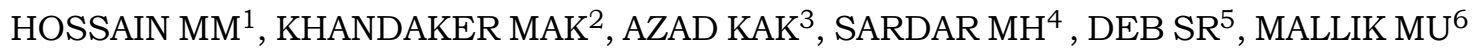

\begin{abstract}
:
Background: The liver biopsy is considered by many experts to be the most specific diagnostic tool used to assess the nature and severity of liver diseases such as hepatitis C. Liver biopsies are important for many reasons, such as accurate diagnosis or ruling out any coexisting liver diseases, staging and grading the severity of liver disease, treatment decisions, patient and provider reassurance, and as a benchmark for gauging future progression.
\end{abstract}

Methods: In this observational study, 50 patients were recruited from different Medicine units of Dhaka Medical College Hospital, Dhaka, from May, 2008 to June, 2009. After preliminary selection of patients, a specific protocol was followed which include patients' particulars, clinical features and clinical diagnosis, biochemical parameters, radiological and other investigative procedures and finally percutaneous needle biopsy of liver was done. The objective of the study was to establish the correlation of clinical presentation of chronic parenchymal liver disease with histopathological findings and establish liver biopsy as a tool for diagnosis. SPSS version 16.0 was used to analyze the data. Quantitative data were presented in the form of tables and figures. Chi-square test and student $t$ test was done to find out the statistical significance.

Results: Histopathologically chronic liver disease (CLD) was found to be the commonest lesion (pd"0.05), which was 24 (48\%) followed by hepatocellular carcinoma 13(26\%) and secondary deposit 02(4\%). Eleven cases consist of other findings including normal. CLD commonly presented with loss of appetite (82\%), Jaundice (74\%), Weight loss (68\%), hepatic facies (54\%). Hepatocellular carcinoma commonly presented with hepatomegaly (100\%), jaundice (61.84\%), weight loss (76.92\%), and ascites (53.85\%). Hepatomegaly was constant feature (100\%) of all secondary metastasis. Among two cases of secondary carcinoma one (50\%) was adenocarcinoma and another one (50\%) was anaplastic type. Out of 29 cases of clinically diagnosed chronic liver disease 24 cases were confirmed histopathologically which was statistically significant ( $p d " 0.05)$.

Conclusion: For the establishment of diagnosis and treatment it is mandatory to have a good correlation between clinical features and histopathological finding. Histopathology could detect diseases which were not considered clinically and specific management could only be done depending on histopathology. Therefore, if there is no contraindication, for confirmation of diagnosis liver biopsy still remains the corner stone modality.

J Dhaka Med Coll. 2013; 22(2) : 120-124.

\section{Introduction}

The liver biopsy is considered by many experts to be the most specific diagnostic tool used to assess the nature and severity of liver diseases such as hepatitis C. Liver biopsies are important for many reasons, such as accurate diagnosis or ruling out any coexisting liver diseases, staging and grading the severity of liver disease, treatment decisions, patient and provider reassurance, and as a benchmark for gauging future progression. There are many different types of liver biopsies, such as the percutaneous, transjugular, laparoscopic, fineneedle aspiration and open surgery liver

1. Dr. Mohammad Murad Hossain, Assistant Professor of Medicine, Dhaka Medical College, Dhaka.

2. Prof. Md. Abul Kashem Khandaker, Professor of Medicine, Popular Medical College, Dhaka.

3. Prof. Khan Abul Kalam Azad, Professor of Medicine, Dhaka Medical College, Dhaka.

4. Dr. Md. Hafiz Sardar, Associate Professor of Medicine, Dhaka Medical College, Dhaka.

5. Dr. Shudip Ranjan Deb, Assistant Professor of Medicine, Dhaka Medical College, Dhaka.

6. Dr. Md. Uzzwl Mallik, Lecturer, Department of Forensic Medicine, Dhaka Medical College, Dhaka.

Correspondence: Dr. Mohammad Murad Hossain, Assistant Professor,Department of Medicine, Dhaka Medical College . Cell Phone: +8801711112984, Email: mhossaink53@gmail.com 
biopsy. ${ }^{1}$ The prognosis of liver disease depends on early diagnosis, appropriate management in many occasions. Skillful suspicion by the attending physician leads to initial rational diagnosis in most of the cases. Auxiliary investigation facilities involving various types of both invasive and non-invasive methods are essential for the proper diagnosis. Liver biopsy is the only investigation for the confirmatory diagnosis of chronic liver disease in spite of tremendous advancement in diagnostic procedures. $^{2}$ Percutaneous needle biopsy of liver is safe, simple, and valuable method for the diagnostic evaluation of liver disease ${ }^{1}$. The mortality rate is less than $0.02 \%$ when performed by experienced operators. Inspite of various modern investigation procedures, it still remains the most attractive investigation.

\section{Materials and Methods}

This was an observational study. A total of fifty cases suspected to have chronic parenchymal liver disease admitted in different medicine units of Dhaka Medical College \& Hospital from May 2008 to June 2009, were included in this study. The age group of the patients was between 15 to 75 years. Details clinical histories and physical findings were recorded. A specific protocol was followed in cases which include patients' particulars, clinical features and clinical diagnosis, biochemical parameters, radiological and other investigative procedures and finally needle biopsy of liver. After taking informed consent percutaneous liver biopsy was done using Trucut needle. The histopathological examination was done in the department of pathology, Dhaka Medical College \& Hospital, Dhaka, BSMMU and other renowned pathological centers in Dhaka. After the preliminary selection of patients, additional clinical history and examination was performed. The details were recorded using a case record form. SPSS version 16.0 was used to analyze the data. Quantitative data were presented in the form of tables and figures. Chi-square test and student $t$ test was done to find out the statistical significance.

\section{Results}

In this study male were significantly (pd"0.05) more affected than female. Out of 50 patients,
$39(78 \%)$ were male and the remaining $11(22 \%)$ were female with a ratio of 3.5: 1 . The youngest patient was 15 years old boy of disseminated tuberculosis and the oldest one was a case of hepatocellular carcinoma aged 75 years. The predominant age group was 41 to 50 years, followed by $31-40$ years (Fig: I).

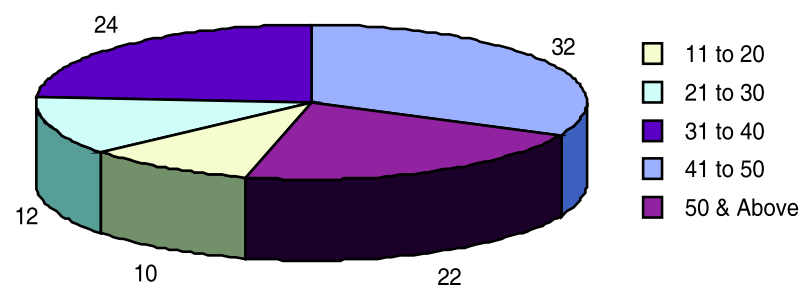

Fig I: Age distribution of studied cases $(N=50)$

Clinical Presentation of fifty cases were studied (Table: I) which included loss of appetite (82\%), Jaundice $(74 \%)$, weight loss $(68 \%)$ as the most common presentations. The next were hepatic facies (54\%), splenomegaly (44\%) and ascites (40\%).

Table -I Clinical presentation of 50 cases of liver
disease

\begin{tabular}{lcc}
\hline Presentation & No. of cases & Percentage(\%) \\
\hline Loss of appetite & 41 & 82 \\
Jaundice & 37 & 74 \\
Weight loss & 34 & 68 \\
Hepatic facies & 27 & 54 \\
Splenomegaly & 22 & 44 \\
Ascites & 20 & 40 \\
Testicular atrophy & 11 & 22 \\
Hepatomegaly & 19 & 38 \\
Fever & 9 & 18 \\
Palmer erythema & 7 & 14 \\
Gynaecomastia & 5 & 10 \\
Loss of libido & 5 & 10 \\
Scanty axillary and & 4 & 8 \\
pubic hair & & 6 \\
Clubbing & 3 & 6 \\
Leukonychia & 3 & 4 \\
Pleural effusion & 2 & \\
\hline
\end{tabular}


Relevant liver function tests were performed and serum bilirubin was found elevated ( $>1 \mathrm{gm} /$ d1) in $39(78 \%)$ cases. Alanine aminotransferase (ALT) was elevated $(>40 \mathrm{U} / 1)$ in 38 $(76 \%)$ cases. Prothrombine time was found prolonged ( $>3$ second from control) in $18(36 \%)$ cases. Serum albumin was reduced $(<3.5 \mathrm{~g} /$ dI) in $20(40 \%)$ cases (Table-II).

Table-II

Routine liver function test ( $n=50)$

\begin{tabular}{lllcc}
\hline Test & \multicolumn{2}{c}{ Normal } & Above normal \\
& No. & $\%$ & No. & $\%$ \\
\hline Serum bilirubin & 11 & 22 & 39 & 78 \\
ALT & 12 & 24 & 38 & 76 \\
Prothrombin time & 32 & 64 & 18 & 36 \\
Serum albumin & 30 & 60 & 20 & 40 \\
\hline
\end{tabular}

Table III described the important serological interpretations. It was found that $\mathrm{HbsAg}$ was positive in $11(68.75 \%)$ cases of cirrhosis, 3 $(42.86 \%)$ cases of chronic hepatitis and 10 $(76.92 \%)$ cases of hepatocellular carcinoma which was statistically significant (p d" 0.05). Anti-HCV was fond in single $(6.2 \%)$ case of cirrhosis. Alpha-feto protein was found elevated in $10(76.92 \%)$ cases of hepatocellular carcinoma (p d" 0.05).

Thirty (58\%) patient presented with features of chronic liver disease. 15 (30\%) of them were proved to be cirrhosis of liver histologically. 16 $(32 \%)$ patient had clinical features of hepatocellular carcinoma, 13 (26\%) of them had hepatocellular carcinoma histologically. Secondary deposit was proved in $02(4 \%)$ cases. Eleven cases consist of other findings including normal.

Table III

Results of important serological tests $(n=50)$

\begin{tabular}{|c|c|c|c|c|c|c|c|}
\hline \multirow[t]{2}{*}{ Diagnosis } & \multicolumn{2}{|c|}{ HBsAg } & \multicolumn{2}{|c|}{ Anti-HCV } & \multicolumn{2}{|c|}{ Alpha-feto protein } & \multirow{2}{*}{$\begin{array}{c}\mathrm{P} \\
\text { value }\end{array}$} \\
\hline & No. & $\%$ & No. & $\%$ & No. & $\%$ & \\
\hline Cirrhosis(N=15) & 10 & 66.67 & 1 & 6.67 & - & - & pd" 0.05 \\
\hline Chronichepatitis $(\mathrm{N}=9)$ & 04 & 44.44 & 0 & 0 & - & - & \\
\hline Hepatocellular carcinoma $(\mathrm{N}=13)$ & 10 & 76.92 & - & - & 10 & 76.92 & \\
\hline Other Diseases & \multicolumn{2}{|c|}{ None } & \multicolumn{2}{|c|}{ None } & \multicolumn{2}{|c|}{ Not done } & \\
\hline
\end{tabular}

Table IV

Clinical correlation with Histopathological finding

\begin{tabular}{|c|c|c|c|}
\hline Clinical Diagnosis & No & Histopathology & No \\
\hline \multirow[t]{7}{*}{ Chronic liver Disease } & \multirow[t]{7}{*}{30} & Cirrhosis & 15 \\
\hline & & Severe chronic hepatitis & 05 \\
\hline & & Minimal chronic hepatitis & 04 \\
\hline & & Normal & 02 \\
\hline & & Kala-azar & 02 \\
\hline & & Fatty change & 01 \\
\hline & & Primary sclerosing cholangitis & 01 \\
\hline \multirow[t]{3}{*}{ Hepatocellular carcinoma } & \multirow[t]{3}{*}{16} & Hepatocellular carcinoma & 13 \\
\hline & & Normal & 02 \\
\hline & & Fatty change & 01 \\
\hline \multirow[t]{3}{*}{ Secondary Carcinoma of liver } & \multirow[t]{3}{*}{4} & Secondary carcinoma of liver & 02 \\
\hline & & Fatty change & 01 \\
\hline & & Normal & 01 \\
\hline
\end{tabular}

Out of the secondaries, one was adenocarcinoma and others had anaplastic type of secondary carcinoma. 


\section{Discussion}

In this study the commonest disease was found to be chronic liver disease (48\%) of which cirrhosis of liver (30\%) was highest followed by severe chronic hepatitis $(10 \%)$, mild chronic hepatitis $(8 \%)$. Next common disease was hepatocellular carcinoma (26\%). Other diseases found were secondary carcinoma of liver $(4 \%)$, fatty change (6\%), kala-azar (4\%), sclerosing cholangitis $(2 \%)$ and histopothological findings revealed normal hepatic tissue in $(10 \%)$ cases. In 500 needles biopsy of liver performed by Anderson revealed cirrhosis of liver $(68.20 \%)$, chronic hepatitis $(7.6 \%)$, acute viral and alcoholic hepatitis $(4.0 \%)$, steatosis $(2.8 \%)$, secondary carcinoma of liver $(2.6 \%)$ and hepatocellular carcinoma $(2.2 \%)^{3}$. In the present study the higher incidence of cirrhosis of liver $(30 \%) \&$ hepatocellular carcinoma $(26 \%)$ is probably due to high prevalence of the disease in Bangladesh, lack of awareness $\&$ due to trends of high prevalence of hepatitis B \& C infection in south East Asian population. ${ }^{4-6}$

In another study done by Dutta revealed that $16(40 \%)$ patient had cirrhosis of liver, $8(20 \%)$ hepatocellular carcinoma with cirrhosis, $8(20 \%)$ chronic active hepatitis and another 8 $(20 \%)$ chronic persistent hepatitis which is also similar to our study. In his study macro-nodular type of cirrhosis was $50 \%$, mixed $43.75 \%$ and micro-nodular in $6.25 \% .^{5}$. In the present study out of 15 cases of cirrhosis of liver, $9(60 \%)$ were macro-nodular type and 6 (40\%) were micronodular. The predominant age group was between $41-50$ years (32\%) followed by (24\%) in the age group of $31-40$ years. The patients of older age group were predominantly affected with cirrhosis of liver, hepatocellular carcinoma, and chronic persistent hepatitis. These observations are consistent with the study of Dutta. ${ }^{5}$ In this study, most of the patients were male $39(78 \%)$ and only $11(22 \%)$ were female with a male to female ratio $3.5: 1$ which were correlated with previous study of Chowdhury. ${ }^{6}$

Commonest clinical presentations were loss of appetite (82\%), Jaundice $(74 \%)$, weight loss (68\%), hepatic facies (54\%), Splenomegaly
(44\%), ascites (40\%) and hepatomegaly (38\%). Dutta $\mathrm{AK}^{5}$ showed weakness, anorexia and swelling of abdomen in $87.5 \%$ cases, where as Hassan $52 \%$ cases and Sabur $42.85 \%$ cases. $^{7,8}$ Jaundice was present in $68.75 \%$ cases of chronic parenchymal liver disease in the study of Dutta, and in $56 \%$ cases in study of Chowdhury. ${ }^{5,6}$ This study revealed that serum bilirubin level was raised in $78 \%$ cases and alanine aminotransferase (ALT) in $76 \%$ cases, prolongation of prothrombin time in $36 \%$ cases, and serum albumin was reduced in $40 \%$ cases which also correlated with the finding of Hassan. ${ }^{7}$ HBsAg was found to be positive in $66.67 \%$ cases of cirrhosis and $76.92 \%$ cases of hepatocellular carcinoma, which was correlated with the recent survey in Iraq where it was showed HBsAg positivity in $58 \%$ of the patients with cirrhosis. Hepatocellular carcinoma is closely associated with chronic $\mathrm{HBV}$ infection. Though it was reported that $\mathrm{HCV}$ causing great number of cases of chronic parenchymal liver diseases but sufficient comparative data is not yet available. Alpha feto protein was found elevated (Significant) in 10 out of 13 cases of hepatocellular carcinoma. Study performed by Hoque revealed raised alpha feto protein level in $58.82 \%$ cases and Chowdhury in $55.55 \%$ cases $^{6,9}$ of Cirrhosis and primary carcinoma of liver is closely related. ${ }^{10}$

Ultrasonogram was done is all cases and showed splenomegaly in $44 \%$ cases, hepatomegaly in $38 \%$ cases and ascites in $40 \%$ cases. But the study conducted by Hassan showed $82 \%$ and by Dutta showed 62.5\%.5,7 Endoscopy of upper gastrointestinal tract revealed oesophageal varices in $40 \%$ cases, which was considerably lower compared to Chowdhury in $83.33 \%$ cases. $^{6}$ In this study incidence of primary carcinoma $(26 \%)$ was more than secondary carcinoma (4\%). There were 13 Patients with hepatocellular carcinoma. Nodular hepatomegaly (100\%), Jaundice $(61.54 \%)$, marked weight loss $(76.92 \%)$ were common presentation of most cases of advanced hepatocellular carcinoma. Only 2 patients of secondary carcinoma of liver were found in this study of which one (1) case were adenocarcinoma and another was anaplastic type. In this study 3 patients had 
fatty change of liver of which two patients were alcoholic and one patient had clinical feature suggestive of malnutrition. Two Cases of leishmaniasis were found incidentally in this study. In visceral leishmaniasis there is massive hypertrophy of kupffer cells and portal phagocytes may contain in variable number of LD bodies. ${ }^{11}$ Parenchymal cell degeneration, and portal tract and sinusoidal infiltration with lymphocytes may be seen. In this study 5 patients had normal histopathological findings. Normal histopathological finding may not exclude presence of cirrhosis in an individual. This is because cirrhous tissue is friable and fragmentation of tissue may not give a clear idea about the change or sometime impossible. Normal finding can be explained because in cirrhosis particularly with ascites may be difficult to pierce and a few liver cells may be extracted leaving the fibrous frame work behind. Although cirrhosis involves the whole liver but large areas may appear almost normal. ${ }^{12}$ In this study, histopathological report showed only one case of primary sclerosing cholangitis and clinically it was diagnosed as a case of chronic liver disease. Though histological appearances are not usually diagnostic but helpful in case of small intrahepatic bile duct disease where endoscopic retrograde cholangio pancreatography (ERCP) or magnetic resonance cholangio pancreatography (MRCP) are normal. In this case ultimately Liver biopsy confirmed the disease.

\section{Conclusion}

For the establishment of diagnosis and treatment it is mandatory to have a good correlation between clinical features and histopathological finding. This study although little have shown that histopathology could detect diseases which were not considered clinically and specific management could only be done depending on histpathology. Therefore, if there is no contraindication, for confirmation of diagnosis liver biopsy still remains the core stone modality.

\section{References}

1. Bravo AA, Sheth SG, Chopra S. Liver biopsy. N Engl J Med 2008; 344: 495-500.

2. Romano M, de Sio R. Decision- making model for a non-invasive diagnosis of compensated liver cirrhosis. Ital J Gastroenterol 2004; 25: 1-8.

3. Anderson E, Ridly DS. Liver biopsy and precutaneous cholangiography using posterior approach 500 needle biopsy. Am J Surg 2003; 121: 31-42.

4. Grant A, Neuberger J. Guidelines on the use of liver biopsy in clinical practice. Gut 1999: 45 (Suppl IV): 12-98.

5. Dutta AK. Chronic Parenchymal liver disease clinical, biochemical, imaging and histopathological correlation [Dissertation]. Dhaka: Bangladesh College of Physicians and Surgeons (BCPS); 1991.

6. Chowdhury AW. A Study of HBsAg status of hospital admitted patients of chronic liver disease and its clinicopathological correlation [Dissertation]. Dhaka: Bangladesh College of Physicians and Surgeons (BCPS); 1995.

7. Hassan MM. Clinical Presentation of cirrhosis of liver [Dissertation]. Dhaka: Bangladesh College of Physicians and Surgeons (BCPS); 1989.

8. Sobur MA. A Clinical Study of chronic liver disease [Dissertation]. Dhaka: Bangladesh College of Physicians and Surgeons (BCPS); 1988.

9. Hoque AKMA. Hepatocellular Carcinoma as the complication of HBV infection and Cirrhosis of liver [Dissertation]. Dhaka: Bangladesh College of Physicians and Surgeons (BCPS); 1994.

10. Islam N, Chowdhury NA, Ali SM. Cirrhosis of liver. Bangladesh Med Res Counc Bull 1976; 2: 6-13.

11. Ridley DS. The laboratory diagnosis of Tropical disease with special reference to Britain: review. J Clin Pathol 1974; 27: 435-44.

12. Abdi W, Millan JC, Mezey E. Sampling Variability in Percutaneous liver biopsy. Arch Intern Med 1979; 139(6): 667-9. 